\title{
Assessment of Cloud Screening with Apparent Surface Reflectance in Support of the ICESat-2 Mission
}

\author{
Yuekui Yang ${ }^{1,2}$, Alexander Marshak ${ }^{2}$, Stephen P. Palm ${ }^{2,3}$, \\ Zhuosen Wang ${ }^{4}$, Crystal Schaaf ${ }^{4}$
}

${ }^{1}$ Universities Space Research Association, Columbia, MD.

${ }^{2}$ NASA Goddard Space Flight Center, Greenbelt, MD.

${ }^{3}$ Science Systems and Applications Inc., Lanham, MD

${ }^{4}$ Geography and Environment Department, Boston University, Boston, MA.

\section{POPULAR SUMMARY}

The separation of cloud and clear scenes is usually one of the first steps in satellite data analysis. Before deriving a geophysical product, almost every satellite mission requires a cloud mask to label a scene as either clear or cloudy through a cloud detection procedure. For clear scenes, products such as surface properties may be retrieved; for cloudy scenes, scientist can focus on studying the cloud properties. Hence the quality of cloud detection directly affects the quality of most satellite operational and research products. This is certainly true for the Ice, Cloud, and land Elevation Satellite-2 (ICESat-2), which is the successor to the ICESat-1. As a top priority mission, ICESat-2 will continue to provide measurements of ice sheets and sea ice elevation on a global scale. Studies have shown that clouds can significantly affect the accuracy of the retrieved results. For example, some of the photons (a photon is a basic unit of light) in the laser beam will be scattered by cloud particles on its way. So instead of traveling in a straight line, these photons are scattered sideways and have traveled a longer path. This will result in biases in ice sheet elevation measurements. Hence cloud screening must be done and be done accurately before the retrievals.

Usually, a space-borne lidar system such as ICESat-1, has the advantage of accurately measuring atmospheric layer heights; hence their cloud detection can generally be achieved by examining the layer information. However, unlike ICESat-1, which has an atmosphere channel for cloud and aerosol detection, the ICESat-2 mission utilizes a low pulse energy laser. So the returning signal is relatively weak. At night, good performance on atmospheric layer detection can be expected through signal averaging. During daytime, however, the signal to noise ratio will be especially low due to the solar 
background noise. Under this situation, the ability of ICESat-2 in detecting atmospheric layers is limited.

Luckily, ICESat-2 can measure a quantity called apparent surface reflectance, which represents the part of the laser energy that passes through the atmosphere, reaches the surface and is reflected back to the satellite. If there were nothing between the satellite and the ground, the apparent surface reflectance would be the real surface reflectance. However, since the laser beam has to pass through the atmosphere (some of the photons will be lost during the process), the value of the apparent reflectance is always lower than the real surface reflectance. When clouds are present, the apparent surface reflectance will be even lower. Based on this fact, this paper proposes to use apparent surface reflectance for cloud screening in support of the ICESat-2 mission. The basis of this method is that clouds produce a strong signal by significantly decreasing surface apparent reflectance. We show that depending on the properties of the cloud, apparent surface reflectance decreases $8 \%-17 \%$ for clouds with an optical depth of 0.1 (optical depth is a measure of transparency. The larger it is, the less transparent it becomes) and $57 \%$ - $85 \%$ for clouds with an optical depth 1.0. Data from ICESat-1 are used to demonstrate the feasibility of the method. It is shown that cloud detectability is a function of surface variability. Generally, the smaller the surface variability, the more accurate is cloud detection. Unlike ICESat-1, which used a $1064 \mathrm{~nm}$ laser, ICESat-2 adopts a $532 \mathrm{~nm}$ laser system. With both modeling studies and results from the Moderate Resolution Imaging Spectroradiometer (MODIS), we demonstrate that the variability in apparent surface reflectance is much smaller at the $532 \mathrm{~nm}$ wavelength than that at the $1064 \mathrm{~nm}$. Hence cloud detection with this method will be better for ICESat-2 than for ICESat-1.

ICESat-2 is scheduled for launch in 2016. Once enough ICESat-2 data are accumulated, a threshold data set can be easily developed and this method can be readily applied for cloud screening. 
Assessment of Cloud Screening with Apparent Surface Reflectance in Support of the ICESat-2 Mission

\author{
Yuekui Yang ${ }^{1,2}$, Alexander Marshak ${ }^{2}$, Stephen P. Palm ${ }^{2,3}$, \\ Zhuosen Wang ${ }^{4}$, Crystal Schaaf ${ }^{4}$
}

Submitted to

IEEE Transaction on Geoscience and Remote Sensing

${ }^{1}$ Universities Space Research Association, Columbia, MD.

${ }^{2}$ NASA Goddard Space Flight Center, Greenbelt, MD.

${ }^{3}$ Science Systems and Applications Inc., Lanham, MD

${ }^{4}$ Geography and Environment Department, Boston University, Boston, MA. 


\begin{abstract}
Cloud detection/screening is a fundamental step in satellite data analysis. For the Ice, Cloud, and land Elevation Satellite (ICESat) and its successor ICESat-2, clouds can significantly affect the accuracy of the surface elevation retrievals. This paper proposes a new method for cloud screening in support of the ICESat-2 mission with focus on the polar ice sheet regions. The method utilizes the apparent surface reflectance at the backscattering direction as the cloud screening test. The basis of this method is that clouds produce a strong signal by significantly decreasing surface apparent reflectance. We show that depending on the height and microphysics of the cloud, apparent surface reflectance decreases $8 \%-17 \%$ for clouds with an optical depth of 0.1 and $57 \%-85 \%$ for clouds with an optical depth 1.0. Data from ICESat's $1064 \mathrm{~nm}$ channel is used to demonstrate the feasibility of the method. It is shown that cloud detectability is a function of surface variability. Generally, the smaller the surface variability, the more accurate is cloud detection. Unlike ICESat, which used a $1064 \mathrm{~nm}$ laser, ICESat-2 adopts a $532 \mathrm{~nm}$ photon counting system for its laser altimeter. With both modeling studies and results from the Moderate Resolution Imaging Spectroradiometer (MODIS), we demonstrate that the variability in apparent surface reflectance is much smaller for the $532 \mathrm{~nm}$ channel than that for the $1064 \mathrm{~nm}$ channel. Hence the $532 \mathrm{~nm}$ channel is better suited for cloud screening than the $1064 \mathrm{~nm}$ channel.
\end{abstract}




\section{Introduction}

The separation of cloud and clear scenes is usually one of the first steps in satellite data analysis. Before retrieving a geophysical product, almost every satellite mission requires a cloud mask to label pixels as either clear or cloudy through a cloud detection procedure (e.g. [1][2][3][4]). The quality of cloud detection directly affects the quality of most satellite operational and research products.

The importance of cloud detection and screening is also true for the Ice, Cloud, and land Elevation Satellite (ICESat) and its successor ICESat-2 [5]. Launched in 2003, ICESat was designed to obtain accurate surface elevation measurements on a global scale [6]. Onboard ICESat was the Geoscience Laser Altimeter System (GLAS), which utilized a $1064 \mathrm{~nm}$ lidar with analog detection to obtain the elevation of the underlying terrain. Studies have shown that clouds can significantly affect the retrieved altimetry. Forward scattering by cloud particles increases the photon path length, thus resulting in biases in ice sheet elevation measurements known as atmospheric path delay [7][8][9]. Similar to ICESat, ICESat-2 will continue the global surface elevation measurements with high accuracy, especially over the ice sheets [10]. Compared to ICESat, the significantly improved design of ICESat-2 employees a $532 \mathrm{~nm}$ photon counting lidar system with single photon detectability. As shown in [11], the ICESat-2 surface altimetry will also be affected by clouds and cloud screening must be done before the retrievals.

Compared to passive remote sensing instruments, space-borne lidars have the advantage of accurately measuring layer heights; hence their cloud detection can generally be achieved by examining the layer information. Examples of using this 
method include the Cloud-Aerosol Lidar and Infrared Pathfinder Satellite Observations (CALIPSO) [12] and the ICESat mission [5]. However, unlike ICESat, which has an atmosphere channel for cloud and aerosol detection, the ICESat-2 mission utilizes a high repetition rate, low pulse energy laser. At night, good performance on atmospheric layer detection can be expected through signal averaging. During daytime, however, the signal to noise ratio will be especially low due to the solar background noise [5]. Under this situation, the ability of ICESat-2 in detecting atmospheric layers is limited.

For ICESat and ICESat-2, a bidirectional reflectance factor (BRF) value at the backscattering direction with nadir illumination can be derived from the energy received within the surface range gate. This value is called apparent surface reflectance because it is the product of the two-way transmittance of the atmosphere and the true surface BRF $[13][14]$. We note that the CALIPSO lidar is not suitable for deriving apparent surface reflectance over the ice sheets, because the surface return would saturate the detectors under clear sky or thin cloud situations. Carabajal et al. [14] demonstrated how the information of apparent surface reflectance could be used to constrain a laser altimeter mission design. In this paper, we investigate the potential of apparent surface reflectance in cloud screening over polar ice sheets for the ICESat-2 mission. The remainder of the paper is organized as follows: Section II introduces the rationale of using apparent surface reflectance as a cloud screening test; in Section III, we examine the impact of surface variability on cloud detectability; Section IV uses the ICESat data to demonstrate the potential of this method in cloud detection; In Section V, modeling and the Moderate Resolution Imaging Spectroradiometer (MODIS) observations are used to show the 
apparent surface reflectance differences in the $1064 \mathrm{~nm}$ and $532 \mathrm{~nm}$ channels. Results are summarized in Section VI.

\section{Cloud Impact On Lidar Surface Returns}

Like most remote sensing instruments, the detectors onboard ICESat and ICESat2 measure the reflected energy into a particular angle. However, unlike passive remote sensing sensors, ICESat and ICESat-2 have time-dependent information and can measure the energy that is reflected by the surface only and reaches the detectors; hence the apparent surface reflectance can be calculated. Similar to the definition of the BRF, the apparent surface reflectance is defined as (e.g. [15]):

$$
\rho_{a p p}\left(\mu_{0}, \mu, \varphi\right)=\frac{\pi L}{\mu_{0} E_{\text {emit }}}
$$

where $L$ is the radiance at the sensor resulted from the photons reflected by the surface; $\mu_{0}$ and $\mu$ are the cosine of illumination zenith angle and view zenith angle, respectively; $\varphi$ is the relative azimuth angle; $E_{\text {emit }}$ is the emitted laser energy. Here, $\mu_{0}=$ $\mu=1.0$. It is easy to see that $\rho_{a p p}$ is the ratio between the measured radiance $L$ and that of a perfect Lambertian reflector (i.e. albedo $=1$ ); hence for a surface with a high albedo, such as fresh snow or ice, $\rho_{a p p}$ can be larger than 1. For ICESat and ICESat-2, the radiance $L$ at the sensor can be expressed as:

$$
L=\frac{E_{r e c} / T_{o p t}}{A_{\text {telescope }} / R^{2}}
$$


where $E_{\text {rec }}$ is the energy that reaches the detectors from the range gate that contains the object (e.g. the surface); $T_{\text {opt }}$ is the transmission of the optics; $A_{\text {telescope }}$ is area of the telescope and $R$ is the distance between the satellite and the surface. Combine Eq. (2) with (1), the apparent reflectance $\rho_{a p p}$ can be derived as [13][14]:

$$
\rho_{\text {app }}=\frac{\pi E_{r e c} R^{2}}{E_{\text {emit }} A_{\text {telescope }} T_{o p t}}
$$

For a given surface and laser system, clouds lower the returning energy that is reflected by the surface $\left(E_{r e c}\right)$; hence lower the apparent surface reflectance. Evidently, when clouds are present, apparent surface reflectance is a function of cloud optical depth (COD), cloud height and cloud microphysical properties. Fig. 1 illustrates how cloud properties affect apparent surface reflectance. The black and gray curves are results of radiative transfer calculations with a 3D Monte Carlo model (validated by the International 3D Radiation Code (I3RC) project [16]). Normalized by the clear sky apparent surface reflectance, the results shown represent the two-way transmittance of the atmosphere. For example, a cloud with $\mathrm{COD}=0.1$ decreases the surface return by about $8 \%$ to $17 \%$; while a cloud with $\mathrm{COD}=1.0$ decreases the surface return by $57 \%$ to $85 \%$. The variability in surface return for a given COD is due to the variability in cloud altitude and microphysics. Fig. 1a shows that the lower the cloud, the higher is the apparent surface reflectance. This is due to the fact that for lower clouds, photons that experienced multiple scattering have a larger chance to stay in the telescope field of view [9]. Similarly, as shown in Fig 1b, everything being equal, the larger the particle size, the higher is the apparent surface reflectance. This is because of their larger forward scattering; hence more photons tend to stay in the FOV. 
The analytical approximations are also shown in Fig 1 with the color curves. For a cloud of optical depth $\tau$, the probability of photons being scattered once and twice are $\tau e^{-\tau}$ and $\frac{\tau^{2}}{2} e^{-\tau}$, respectively (e.g., [7] and [24]). Weighting these quantities with the probability of being scattered into a small forward angle (which is $1 / 2$, based on the diffraction theory) leads to the following simple approximations for the two-way transmittance when zero, first and second scattering orders are taken into account, respectively:

$$
\begin{aligned}
& T_{0}(\tau)=e^{(-2 \tau)} \\
& T_{1}(\tau)=e^{(-2 \tau)}(1+\tau / 2)^{2} \\
& T_{2}(\tau)=e^{(-2 \tau)}\left(1+\tau / 2+\tau^{2} / 8\right)^{2}
\end{aligned}
$$

Obviously, as an approximation, the analytical results do not depend on cloud height, geometrical thickness, microphysics or the telescope FOV, but compared to the time consuming Monte Carlo simulations, when up to second order scatterings are accounted, the analytical approximations provide reasonable accuracy with minimal computational effort. Overall, to summarize the results of Fig. 1, clouds provide a strong signal in apparent surface reflectance that can be used for cloud detection.

\section{Apparent Surface Reflectance Distribution And Cloud Detectability}

Section II shows that clouds can significantly reduce apparent surface reflectance measured by the ICESat-2 detectors; hence it is possible to set a threshold to separate cloudy and clear conditions. However, if surface reflectivity varies significantly from location to location, cloud detectability will be lower compared to an otherwise uniform 
surface. To show this, we first examine how apparent surface reflectance varies under clear sky conditions over the ice sheets. Fig. 2a gives the results over Greenland, East and West Antarctica. Data are from the GLAS L2A campaign that began on 25 September and lasted until 19 November, 2003 [17]. All data for which clouds were detected have been removed. We selected the L2A campaign because during this period the GLAS had a fully functional atmosphere channel and the best cloud detection ability [18]. As mentioned before, the apparent surface reflectance was derived from the $1064 \mathrm{~nm}$ channel measurement. Since aerosols are generally optically thin and Rayleigh scattering is negligible at the $1064 \mathrm{~nm}$, the apparent surface reflectance under clear sky is very close to the surface BRF and its distribution represents the variability of the surface reflectivity. It can be seen from the figure that the mode of the distribution from the Greenland ice sheet is lower than that of the Antarctica regions, with the highest from the East Antarctica. As explained in Section II, notice that some of the apparent reflectance values are larger than one.

The distributions shown in Fig. 2a resemble a bell shape (Gaussian). To better illustrate the impact of surface variability on cloud detection, we first fit the clear sky distributions with a Gaussian function. The best fit for the distributions of Greenland, East and West Antarctica regions has a mean of $0.87,0.91$ and 0.89 and a standard deviation of $0.07,0.07$ and 0.06 , respectively. Fig. $2 b$ uses the Gaussian fit to the East Antarctica distribution (solid black line) as an idealized apparent surface reflectance of snow cover under clear sky conditions. When clouds are present, the apparent reflectance will be smaller. If we assume cloud is a plane parallel layer, the shape of the distribution curve will not change; only the mean becomes smaller. The result is that the entire curve 
is shifted to the left. The size of the shift is a function of the cloud properties. For example, based on the radiative transfer simulations conducted in Section II, for a cloud at 0.5 to $1.0 \mathrm{~km}$ with MODIS ice phase function for $\mathrm{r}_{\mathrm{e}}=20 \mu \mathrm{m}$, apparent reflectance would decrease by $10 \%$ for $\mathrm{COD}=0.1$ and $41 \%$ for $\mathrm{COD}=0.5$. This example is plotted in Fig. $2 b$ with the dashed lines.

As can be seen in Fig. 2b, there is an overlap between the apparent reflectance distributions for clear sky and for cloudy sky (e.g. the curve for COD =0.1). Hence it is no longer possible to completely separate clear and cloudy pixels with a single threshold [19]: no matter where the threshold is set, some of the pixels will be misclassified. Generally, a smaller standard deviation would indicate less overlap between the distributions under clear and cloudy sky conditions; hence better cloud detection. Fig. $2 b$ and $2 \mathrm{c}$ demonstrate this point. The Gaussian distributions for clear sky in both panels have the same mean (0.91), yet their standard deviations are different, one is 0.07 (Fig. $2 b$ ) and the other 0.03 (Fig 2c). As can be seen, the case with smaller standard deviation (Fig. 2c) corresponds to a smaller overlap, thus better cloud detection.

Evidently, for a given distribution of apparent surface reflectance, the misclassification rate is a function of the threshold and cloud optical depth. Fig. 3 shows the results for the cases used in Fig. 2b and Fig. 2c. For example, for the case with standard deviation of 0.07 (Fig. 3a), if the threshold is set at $0.86,28 \%$ of the clouds with $\mathrm{COD}=0.1$ will be misclassified as clear; the number drops to $6 \%$ and $0.5 \%$ for $\mathrm{COD}=$ 0.2 and 0.3 , respectively; the misclassification for clouds thicker than 0.2 is essentially negligible. Also, part of the clear pixels (23\%) is misclassified as cloudy. For the case with standard deviation of 0.03 (Fig 3b), the same threshold would result in $8 \%$ of the 
clouds with $\mathrm{COD}=0.1$ being misclassified as cloudy and misclassification for optically thicker clouds are negligible. The misclassification for clear pixels is about $5 \%$.

\section{Applications To the ICESat Data}

In this section, we apply the apparent surface reflectance cloud detection method to ICESat data to investigate its feasibility. Again, the $1064 \mathrm{~nm}$ channel data from the GLAS L2A campaign is used. As mentioned in the previous section, for the L2A campaign, accurate cloud detection was achieved from the atmospheric channel; hence we know the "truth" of which pixel is clear and which one is cloudy. The apparent surface reflectance distributions under clear and cloudy sky conditions are built from all the clear and cloudy pixels, respectively.

Fig. 4 shows the results for West Antarctica. As shown in the figure, an overlap exists between the distributions for clear and cloudy sky conditions; hence no threshold could separate all the clear pixels from the cloudy ones. Actually, as pointed out in [19], for a threshold to work perfectly, the histogram of all the pixels has to be discontinuous, with clear pixels on one side and cloudy pixels on the other, yet this type of histogram has never been observed in satellite remote sensing. The bimodal distribution shown in Fig. 4 indicates that apparent reflectance is a good test for cloud detection because misclassification can be minimized by putting the threshold in the valley region of the histogram [4]. A sample threshold of 0.7 is marked on Fig. 4. With this threshold, $15 \%$ of the clear pixels are misclassified as cloudy and $8 \%$ of the cloudy misclassified as clear. Certainly the threshold can be adjusted to make the results either more cloud or clear conservative. As will be discussed in Section V, for the ICESat-2 mission, the $532 \mathrm{~nm}$ 
laser will be used and cloud detectability will be strongly enhanced with the apparent reflectance method.

\section{Apparent Surface Reflectance at the $532 \mathrm{~nm}$ Wavelength}

The observations shown in Section III and IV are all from ICESat's $1064 \mathrm{~nm}$ lidar. Since ICESat-2 will make measurements at the $532 \mathrm{~nm}$, it would be more instructive to look at how apparent surface reflectance behaves at this wavelength. However, at $532 \mathrm{~nm}$, direct laser measurements on surface reflectance over the ice sheets are rare. As mentioned above, space-borne lidars designed for atmospheric measurements, such as the one onboard CALIPSO and the ICESat atmospheric channel lidar, are not suitable for this purpose, because the surface signal saturates the detectors when the scene is clear or covered with optically thin clouds.

Since we need data for nadir illumination to investigate the problem, most passive remote sensing instruments are not helpful either because of the low solar angle over the Polar regions, even though they may have the $532 \mathrm{~nm}$ channel. However, the MODIS surface Bidirectional Reflectance Distribution Function (BRDF) model parameters product may shed some light on this issue. The product is derived based on a kerneldriven semi-empirical model that uses the RossThick-LiSparse kernel functions at a spatial resolution of $1 \mathrm{~km}$ by inverting MODIS observations in a 16-day period (Baseline inversion occurs at $500 \mathrm{~m}$ resolution; the $1 \mathrm{~km}$ resolution product is an average of the underlying 500m data. For details, see [20]). The time interval for the product is eight 
days. The surface BRF with nadir illumination and nadir view angle can be calculated with the parameters provided [20].

Fig. 5a shows the surface BRF distributions with nadir illumination and nadir view angle for four MODIS channels at the $469 \mathrm{~nm}, 555 \mathrm{~nm}, 645 \mathrm{~nm}$ and $858.5 \mathrm{~nm}$ wavelengths. The $555 \mathrm{~nm}$ channel is fairly close to the $532 \mathrm{~nm}$ channel, but unfortunately, MODIS does not have the $1064 \mathrm{~nm}$ channel. The closest approximation is the $858.5 \mathrm{~nm}$ channel. As can be seen from the figure, for these channels, the shorter the wavelengths, the larger the BRF values. Fig. $5 \mathrm{~b}$ compares the mean and standard deviation of the BRF of the $858.5 \mathrm{~nm}$ and the $555 \mathrm{~nm}$ channels for each of the 16-day periods during the winter months of 2008. As shown in the figure, not only is the BRF of the green channel larger $(0.95-097$ at the $555 \mathrm{~nm}$ vs. $0.84-0.88$ at the $858.5 \mathrm{~nm})$, the magnitudes of its standard deviation is also substantially lower $(0.011-0.017$ at the 555 $\mathrm{nm}$ vs $0.018-0.025$ at the $858.5 \mathrm{~nm}$ ). As a result, cloud detectability will be better with the $555 \mathrm{~nm}$ channel.

Another way of looking at how $532 \mathrm{~nm}$ channel values would differ from the $1064 \mathrm{~nm}$ channel values is to convert the ICESat $1064 \mathrm{~nm}$ observations to $532 \mathrm{~nm}$ values through radiative transfer modeling. Here we adopt the analytical asymptotic radiative transfer (AART) snow model developed by Kokhanovsky and Zege [21], in which surface BRF can be approximated as:

$$
\begin{aligned}
& B R F\left(\mu_{0}, \mu, \varphi\right)=R_{0}\left(\mu_{0}, \mu, \varphi\right) \exp \left(-A\left(\mu_{0}, \mu, \varphi\right) \sqrt{\gamma d}\right) \\
& A\left(\mu_{0}, \mu, \varphi\right) \cong 0.66\left(1+2 \mu_{0}\right)(1+2 \mu) / R_{0}\left(\mu_{0}, \mu, \varphi\right) \\
& \gamma=4 \pi \chi / \lambda
\end{aligned}
$$


where $\mu_{0}$ and $\mu$ are the cosine of illumination zenith angle and view zenith angle; $\varphi$ is the relative azimuth angle; $\lambda$ is the wavelength; $\chi$ is imaginary part of the refractive index and $\mathrm{R}_{0}$ is the radiative transfer solution of $\mathrm{BRF}$ for semi-infinite media with no absorption. In this study $\mathrm{R}_{0}$ is calculated with the model developed by Mishchenko et al. [22].

Since aerosols are generally optically thin over ice sheets and Rayleigh scattering is negligible at $1064 \mathrm{~nm}$, the apparent surface reflectance at this wavelength can be considered as virtually the same as the surface BRF. Therefore to obtain the apparent surface reflectance at $532 \mathrm{~nm}$, we first convert the $1064 \mathrm{~nm}$ ICESat observations (surrogate surface BRFs) to the $532 \mathrm{~nm}$ values with the AART model by assuming the variability is only caused by snow grain size. Warren et al. [23] summarized the factors that affect snow surface BRDF, which are: snow grain size, single scattering phase function, illumination zenith angle, absorption coefficient (a function of wavelength), and surface roughness. For the problem we are looking at, the illumination angle and the absorption coefficient do not change. Particle phase function is determined by particle size and shape, but mostly particle size. Surface roughness does change from place to place, but as pointed out by Warren et al. [23], for nadir illumination, its effect is less significant compared to oblique sun angles. Hence the assumption that surface BRF variability is caused by snow grain size can be adopted as a first order approximation. After obtaining the modeled surface BRF, the apparent reflectance (top of atmosphere $\mathrm{BRF}$ ) is calculated by taking into account of the Rayleigh scattering, which is much more significant at the $532 \mathrm{~nm}$ than at the $1064 \mathrm{~nm}$ wavelength. 
Fig. 6 compares the modeled $532 \mathrm{~nm}$ BRF distribution with the $1064 \mathrm{~nm}$ one used in Fig. 2b. As can be seen from the figure; corresponding to a wide range of BRF values at the $1064 \mathrm{~nm}$ (from 0.6 to 1.1 ), the $\mathrm{BRF}$ at the $532 \mathrm{~nm}$ varies much less (from 1.11 to 1.15). Compared to the $1064 \mathrm{~nm}$ standard deviation (0.07), the simulated $532 \mathrm{~nm}$ result is much smaller (0.004). The figure also shows the effect of Rayleigh scattering. While the distribution of the $1064 \mathrm{~nm}$ results do not change much with and without the consideration of Rayleigh scattering, the $532 \mathrm{~nm}$ results change significantly. The mean changed from 1.14 at the surface to 0.90 at the top of atmosphere.

We notice that there are certainly differences in $532 \mathrm{~nm}$ BRF distributions from the AART model and the 555nm distributions from the MODIS observations. These differences can come from multiple sources. For example, (1) the resolution difference between the MODIS retrieval $(1 \mathrm{~km})$ and the ICESat dataset used here (averaged over 7 $\mathrm{km}$ ) may play an important role; (2) the modeling results are based on the assumption that surface BRF variability is caused by snow grain size only, which certainly has its limitations; (3) the MODIS retrievals over the polar regions has uncertainties as well (e.g.

due to the very low solar zenith angle and the residual cloud cover). We are investigating the contribution of the above mentioned factors, but the point we want to make here is that both results show that the $532 \mathrm{~nm}$ channel has a smaller standard deviation; hence it is better suited for cloud screening.

\section{Summary}

ICESat-2 is a top priority NASA mission. Unlike its predecessor ICESat, which used a $1064 \mathrm{~nm}$ lidar for surface altimetry and had a separate lidar system for 
atmospheric measurements, ICESat- 2 adopts a high repetition rate $532 \mathrm{~nm}$ photon counting system. It is more challenging for ICESat-2 to detect clouds, especially in sunlit conditions. This paper proposes a new method of using apparent surface reflectance for cloud screening in support of the ICESat-2 mission. Major results can be summarized as follows:

(1) Clouds produce a strong signal in apparent surface reflectance. It is shown that depending on cloud height and microphysics, apparent surface reflectance may be decreased by $8 \%-17 \%$ for $\mathrm{COD}=0.1$ and $57 \%-85 \%$ for $\mathrm{COD}=1.0$. A new analytical approximation that accounts for contributions from the first and second order scattering has been derived (Eq. (5) and (6)) and compared with the Monte Carlo calculations.

(2) The apparent surface reflectance method shows reasonable results when applied to the GLAS data. For example, over the West Antarctica region, a threshold of 0.7 can be used to detect clouds. In this case, $15 \%$ of the clear pixels are misclassified as cloudy and $8 \%$ of the cloudy pixles are misclassified as clear. The threshold can be adjusted to achieve more cloud or clear conservative results.

(3) Cloud detectability is a function of surface variability. Generally, the smaller the surface variability, the more accurate is cloud detection. Both modeling studies and MODIS results show that the variability in apparent surface reflectance is much smaller for the $532 \mathrm{~nm}$ channel than that of the $1064 \mathrm{~nm}$ channel; hence better cloud screening results can be achieved with the future ICEsat- 2 mission.

ICESat-2 is scheduled for launch in 2016. Once enough ICESat-2 data are accumulated, a threshold data set can be easily developed and this method can be readily applied for cloud screening. 
Acknowledgements: This work was supported by the National Aeronautics and Space Administration's ICESat-2 Science Definition Project.

References

[1] Rossow, W.B. and L.C. Garder, 1993. Cloud Detection Using Satellite Measurements of Infrared and Visible Radiances for ISCCP. Journal of Climate, 6(12): 2341-2369.

[2] Ackerman, S.A., K.I. Strabala, W.P. Menzel, R.A. Frey, C.C. Moeller and L.E. Gumley, 1998. Discriminating clear sky from clouds with MODIS. Journal Of Geophysical Research-Atmospheres, 103(D24): 32141-32157.

[3] Stowe, L.L., P.A. Davis and E.P. McClain, 1999. Scientific Basis and Initial Evaluation of the CLAVR-1 Global Clear/Cloud Classification Algorithm for the Advanced Very High Resolution Radiometer. Journal of Atmospheric and Oceanic Technology, 16(6): 656-681.

[4] Yang, Y., L. Di Girolamo and D. Mazzoni, "Selection of the automated thresholding algorithm for the Multi-angle Imaging SpectroRadiometer Radiometric Camera-byCamera Cloud Mask over land," Remote Sensing Of Environment, vol. 107, pp. 159$171,2007$.

[5] Palm, S. P., J. D. Spinhirne, W. D. Hart, and D. L. Hlavka, 2002: GLAS atmospheric data products, algorithm theoretical basis document, version 4.2, GSFC, Greenbelt, Md. (Available at: http://www.csr.utexas.edu/glas/pdf/glasatmos.atbdv4.2.pdf.). 
[6] Zwally H.J., B. Schutz, W. Abdalati,J. Abshire, C. Bentley, A. Brenner, J. Bufton, J. Dezio, D. Hancock, D. Harding, T. Herring, B. Minster, K. Quinn, S. Palm, J. Spinhirne, and R. Thomas, "ICESat 's laser measurements of polar ice, atmosphere, ocean, and land," J. Geodynamics, vo. 34, pp. 405-445, 2002.

[7] Duda, D.P., J. D. Spinhirne, and E.W. Eloranta, "Atmospheric multiple scattering effects on GLAS altimetry - part I: Calculations of single path bias," IEEE Trans. Geos. Rem. Sens., vol. 39, pp. 92-101, 2001.

[8] Mahesh, A., J. D. Spinhirne, D.P. Duda, and E.W. Eloranta, "Atmospheric multiple scattering effects on GLAS altimetry - part II: Analysis of expected errors in Antarctic altitude measurements," IEEE Trans. Geos. Rem. Sens., vol. 40, pp. 2353$2362,2002$.

[9] Yang, Y., A. Marshak, T. Varnai, W. Wiscombe, P. Yang, "Uncertainties in IceSheet Altimetry From a Spaceborne 1064-nm Single-Channel Lidar Due to Undetected Thin Clouds," IEEE Trans. Geos. Remote Sens., vol. 48, 250-259. 2010.

[10] Abdalati, W.; Zwally, H.J.; Bindschadler, R.; Csatho, B.; Farrell, S.L.; Fricker, H.A.; Harding, D.; Kwok, R.; Lefsky, M.; Markus, T.; Marshak, A.; Neumann, T.; Palm, S.; Schutz, B.; Smith, B.; Spinhirne, J.; Webb, C.; , "The ICESat-2 Laser Altimetry Mission," Proceedings of the IEEE, vol.98, no.5, pp.735-751, May 2010, doi: 10.1109/JPROC.2009.2034765.

[11] Yang, Y., A. Marshak, S. PALM T. Varnai, W. Wiscombe, 2011: Cloud Impact On Surface Altimetry From A Spaceborne 532 nm Micro-Pulse Photon Counting Lidar: 
System Modeling For Cloudy And Clear Atmospheres. IEEE Trans. Geos. Remote Sens. doi: 10.1109/TGRS.2011.2153860.

[12] Vaughan, M., K. Powell, R. Kuehn, S. Young, D. Winker, C. Hostetler, W. Hunt, Z. Liu, M. McGill, B. Getzewich, 2009: "Fully Automated Detection of Cloud and Aerosol Layers in the CALIPSO Lidar Measurements", J. Atmos. Oceanic Technol., 26, 2034-2050, doi:10.1175/2009JTECHA1228.1.

[13] A. C. Brenner, H. J. Zwally, C. R. Bentley, B. M. Csatho, D. J. Harding, M. A. Hofton, J. B. Minster, L. A. Roberts, J. L. Saba, R. H. Thomas, and D. Yi, "Geoscience laser altimeter system algorithm theoretical basis document: Derivation of range and range distributions from laser pulse waveform analysis," Algorithm Theoretical Basis Documents (ATBD), 2003. [Online]. Available: http://www.csr.utexas.edu/glas/atbd.html.

[14] Carabajal, C. C., D. J. Harding, C. A. Shuman, and V. P. Suchdeo, "Laser Altimeter Mission Design Constraints from ICESat Observations of $1064 \mathrm{~nm}$ Apparent Surface Reflectance", American Geophysical Union Fall Meeting, San Francisco, CA, USA, 10-14 December 2007.

[15] Nicodemus, F. E., Richmond, J. C., Hsia, J. J., Ginsberg, I. W., and Limperis, T.: Geometrical Considerations and Nomenclature for Reflectance, NBS Monogr., vol.160, Natl. Inst. of Stand. And Technol., Gaithersburg, Md, 1977.

[16] Cahalan R. F. et al., "The International Intercomparison of 3D Radiation Codes (I3RC): Bringing together the most advanced radiative transfer tools for cloudy atmospheres," Bulletin Amer. Meteor. Soc. (BAMS), vol. 86, pp. 1275-1293, 2005. 
[17] Yang, Y., A. Marshak, J. C. Chiu, W. J. Wiscombe, S. P. Palm, A. B. Davis, D. A. Spangenberg, L. Nguyen, J. D. Spinhirne, and P. Minnis, "Retrievals of Thick Cloud Optical Depth from the Geoscience Laser Altimeter System (GLAS) by Calibration of Solar Background Signal," J. Atmos. Sci., vol. 65, pp. 3513-3527, 2008.

[18] Spinhirne, J.D., S.P. Palm, W.D. Hart, D.L. Hlavka, and E.J. Welton, 2005b: Cloud and aerosol measurements from GLAS: Overview and initial results. Geophys. Res. Letters, 32 (22), L22S03, doi:10.1029/2005GL023507.

[19] Yang, Y., and L. Di Girolamo, "Impacts of 3-D radiative effects on satellite cloud detection and their consequences on cloud fraction and aerosol optical depth retrievals,"J. Geophys. Res., vol. 113, 2008, D04213, doi:10.1029/2007JD009095.

[20] Schaaf, C. B., F. Gao, A. H. Strahler, W. Lucht, X. Li, T. Tsang, N. C. Strugnell, X. Zhang, Y. Jin, J.-P. Muller, P. Lewis, M. Barnsley, P. Hobson, M. Disney, G. Roberts, M. Dunderdale, C. Doll, R. d'Entremont, B. Hu, S. Liang, and J. L. Privette, and D. P. Roy, Operational BRDF, Albedo and Nadir Reflectance Products from MODIS, Remote Sens. Environ., 83, 135-148, 2002.

[21] Kokhanovsky, A., \& Zege, E. P. (2004). Scattering optics of snow. Applied Optics, $43,1589-1602$.

[22] Mishchenko, M.I., J.M. Dlugach, E.G. Yanovitskij, and N.T. Zakharova, 1999: Bidirectional reflectance of flat, optically thick particulate layers: An efficient radiative transfer solution and applications to snow and soil surfaces. J. Quant. Spectrosc. Radiat. Transfer, 63, 409-432, doi:10.1016/S0022-4073(99)00028-X. 
[23] Warren, S. G., R. E. Brandt, and P. O'Rawe Hinton (1998), Effect of surface roughness on bidirectional reflectance of Antarctic snow, J. Geophys. Res., 103(E11), $25,789-25,807$.

[24] Thomas, G. E., and K. Stamnes, 1999: Radiative transfer in the atmosphere and ocean. Cambridge University Press, 517 pp. 

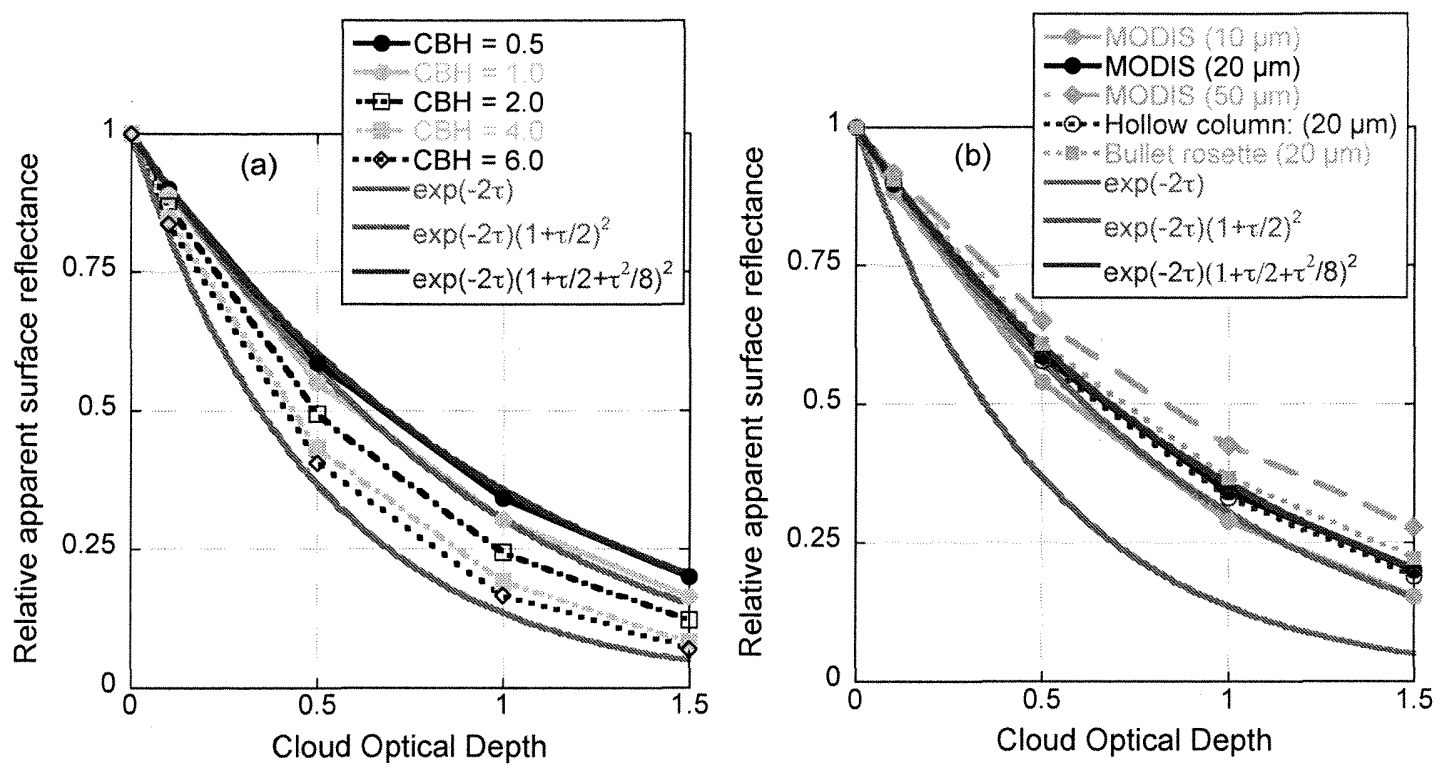

Fig. 1. The effect of cloud properties on ICESat-2 apparent surface reflectance. Results are normalized by the clear sky apparent surface reflectance. Black and gray curves are from Monte Carlo simulations and the color curves are analytical approximations from the radiative transfer and the diffraction theories. Red is for direct transmittance; green is the case when only single order scattering is accounted; blue is when both the first and second order scatterings are accounted. For the Monte Carlo simulations, Telescope of field of view (FOV) is $40 \mathrm{~m}$ in diameter. (a) Apparent surface reflectance changes as a function of COD for different cloud base heights ( $\mathrm{CBH}$ ) (in $\mathrm{km}$ ). Cloud thickness is assumed to be $0.5 \mathrm{~km}$; MODIS ice phase function for particle effective radius $\mathrm{r}_{\mathrm{e}}=20 \mu \mathrm{m}$ is used. Note that the blue curve and the $\mathrm{CBH}=0.5$ curve are overlapping each other; so are the green curve and $\mathrm{CBH}=1.0$. (b) Same as (a) but for different cloud particle sizes and shapes. The numbers in the parentheses in the legend are the effective radii. Clouds are assumed to be located at $0.5-1.0 \mathrm{~km}$. Note that the three curves for $\mathrm{r}_{\mathrm{e}}=20 \mu \mathrm{m}$ for different particle shapes are very close to each other. 

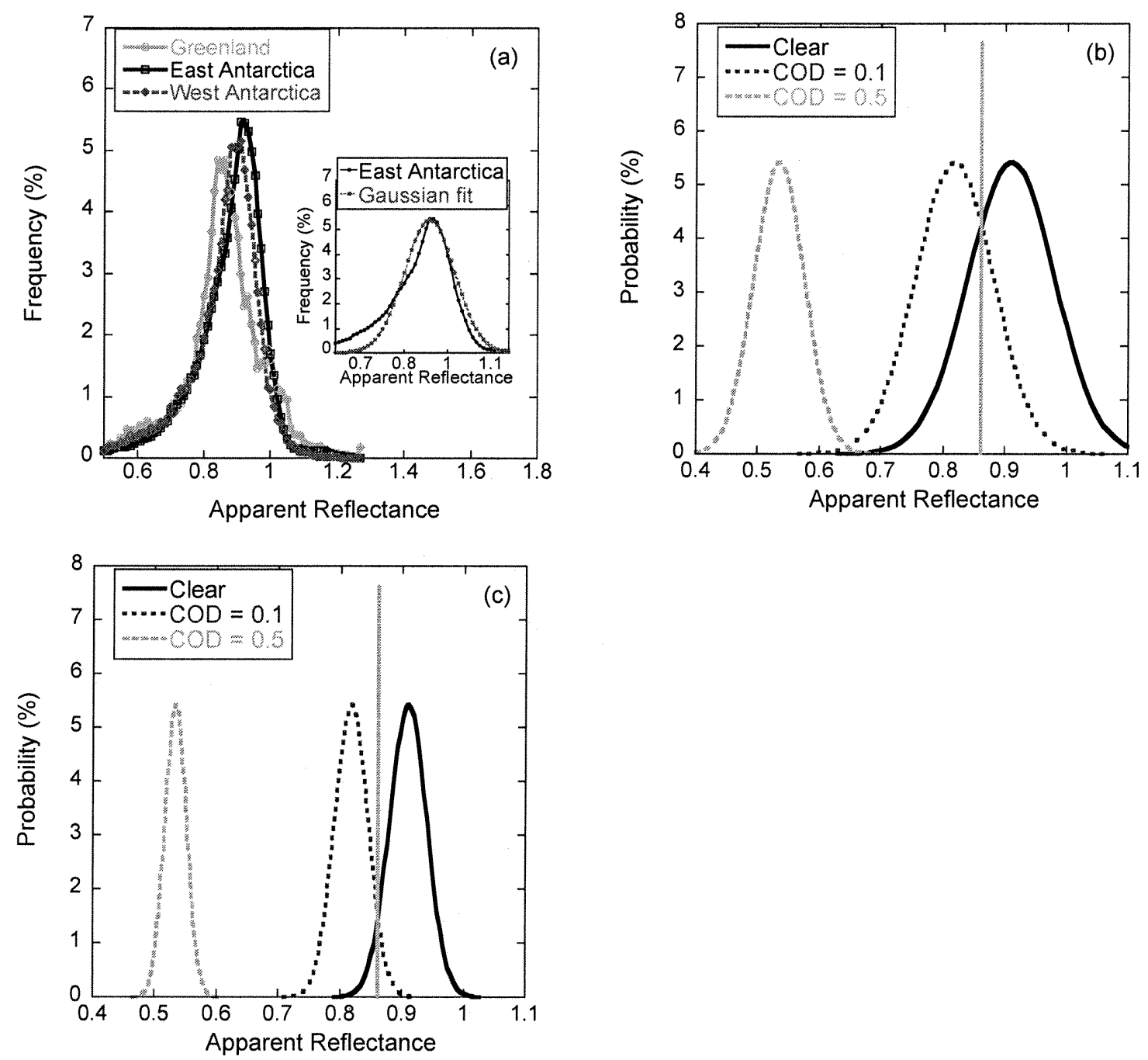

Fig. 2. (a) Distribution of $1064 \mathrm{~nm}$ channel clear sky apparent surface reflectance. The inset is the Gaussian fit for the distribution over the East Antarctica. Data are from the GLAS L2A campaign. (b) Idealized apparent reflectance distribution (Gaussian) for clear sky (mean $=0.91$; standard deviation $=0.07$ ) (solid black line), and the corresponding distributions for $\mathrm{COD}=0.1$ (dashed black line) and $\mathrm{COD}=0.5$ (dashed grey line). Also shown is an example threshold (solid gray line). (c) Same as (b), but for a Gaussian distribution with mean $=0.91$ and standard deviation $=0.03$. 

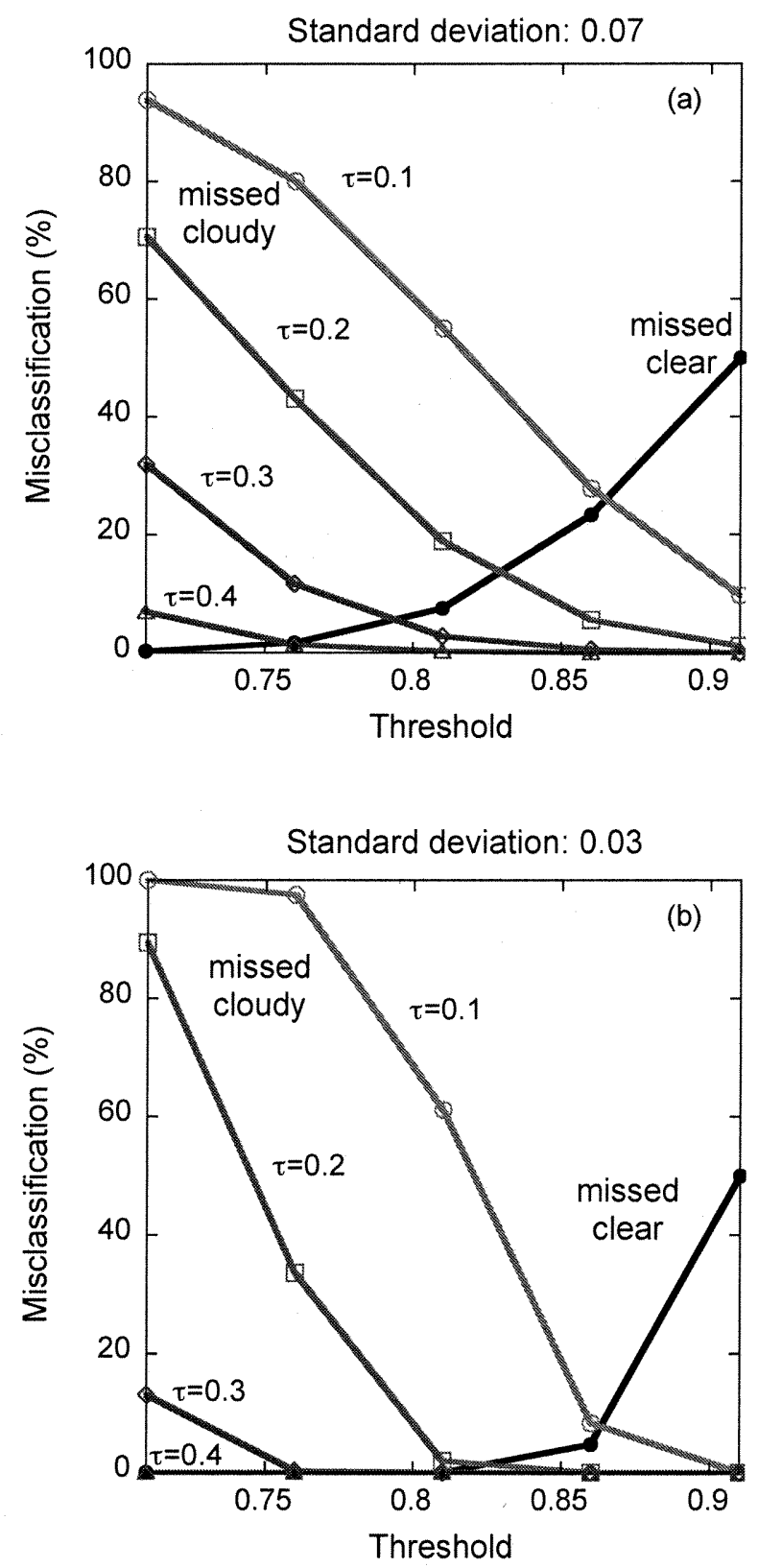

Fig. 3. Cloud misclassification rate as a function of threshold for different cloud optical depth $\tau$. Also shown is the misclassification rate of clear pixels (black line). Clear sky apparent surface reflectance is assumed Gaussian with mean $=0.91$. (a) For the distribution with a standard deviation of 0.07 ; (b) for the distribution with a standard deviation of 0.03 . 


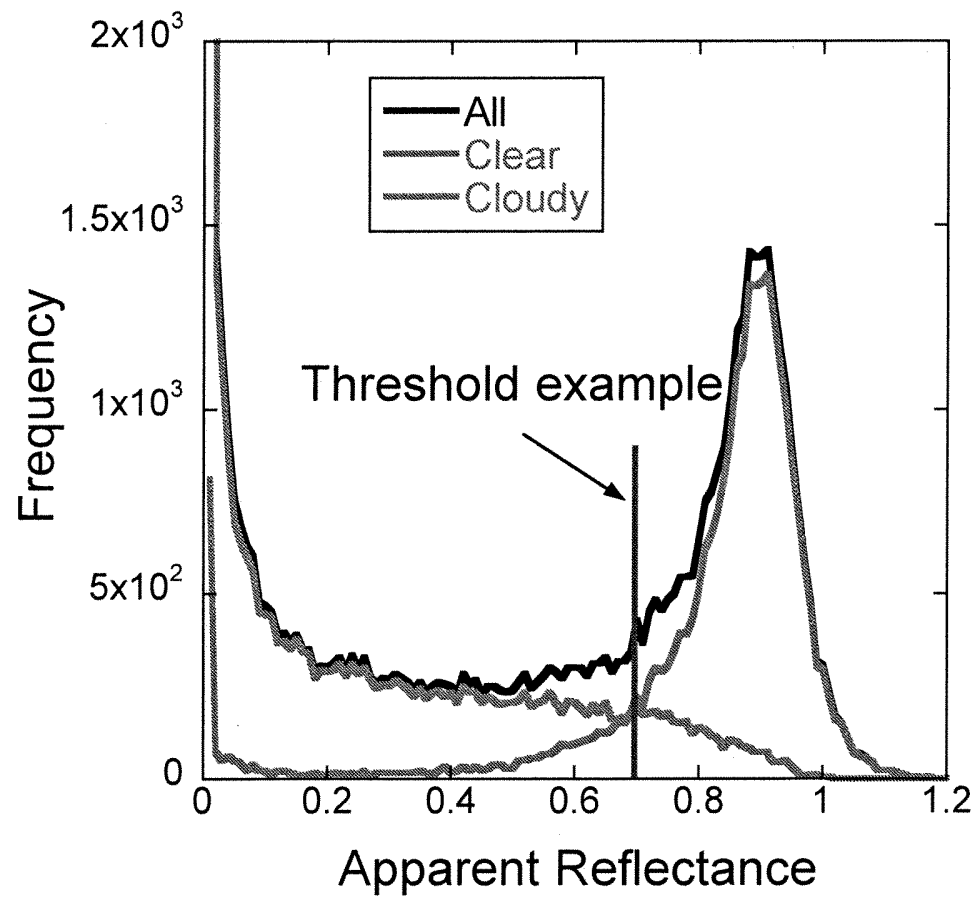

Fig. 4. Apparent surface reflectance distributions for clear (green), cloudy (red) and total (black) sky conditions over the West Antarctica ice sheet. Data are from the GLAS L2A campaign. 

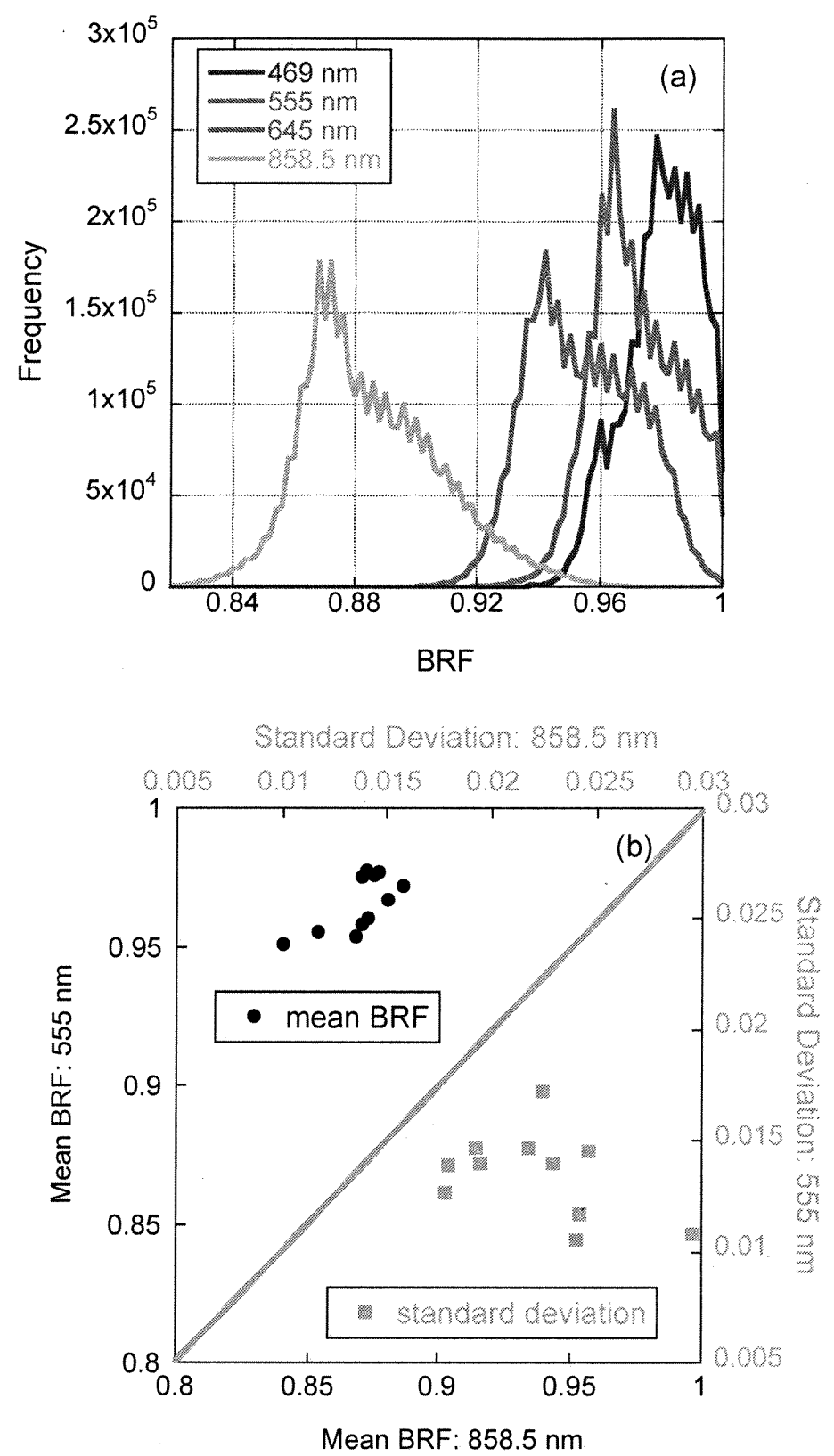

Fig. 5. Interior East Antarctica (73S - 80S, 90E - 145E) surface BRF properties derived from MODIS observations for nadir illumination and nadir view angle. Only data with the best quality flag are used. (a) Surface BRF distributions for four MODIS channels. MODIS observations used are from Jan. 1 to 16, 2008; (b) average BRF and its standard deviation comparison between the $858.5 \mathrm{~nm}$ and the $555 \mathrm{~nm}$ channels. MODIS observations used are from the months of Dec., Jan. and Feb., 2008. Each dot in the figure represents one 16-day period. 


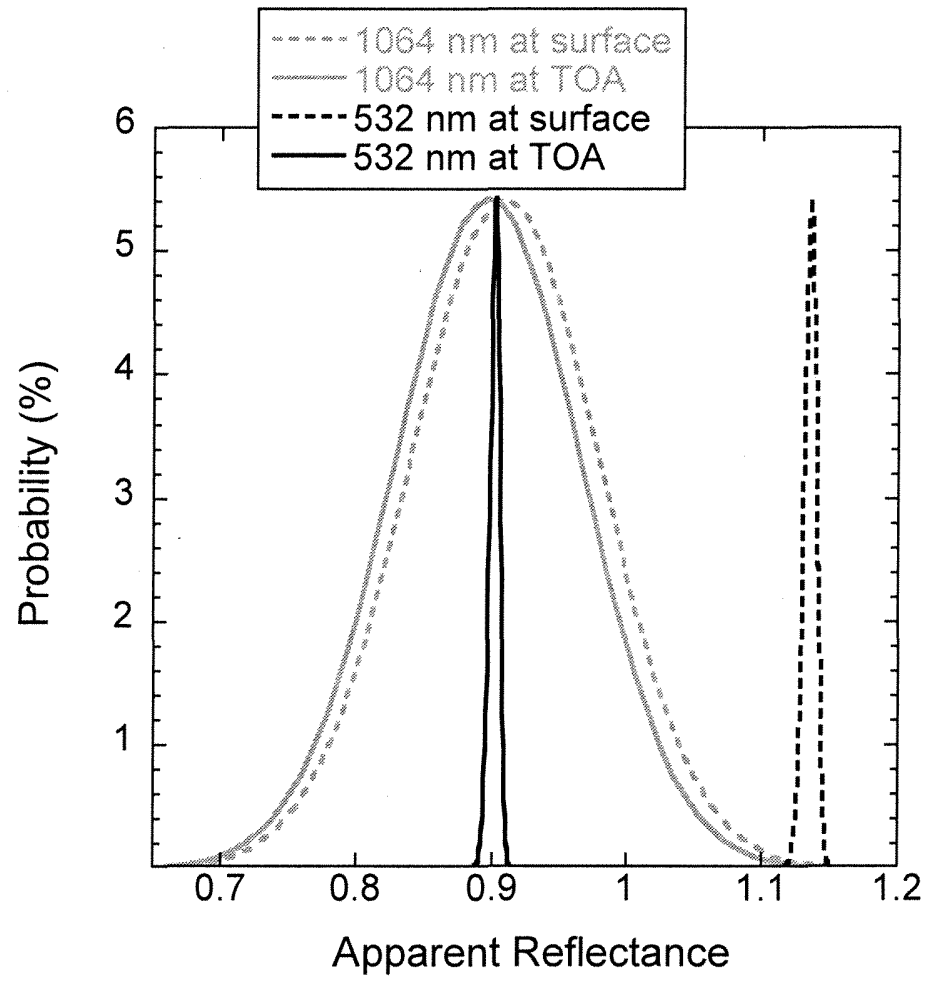

Fig. 6. Snow BRF distributions at $1064 \mathrm{~nm}$ (grey lines) and $532 \mathrm{~nm}$ (black lines) wavelengths. Solid and dashed lines are for results at the top of atmosphere (TOA) and at the surface, respectively. The $532 \mathrm{~nm}$ results are based on the AART model. 